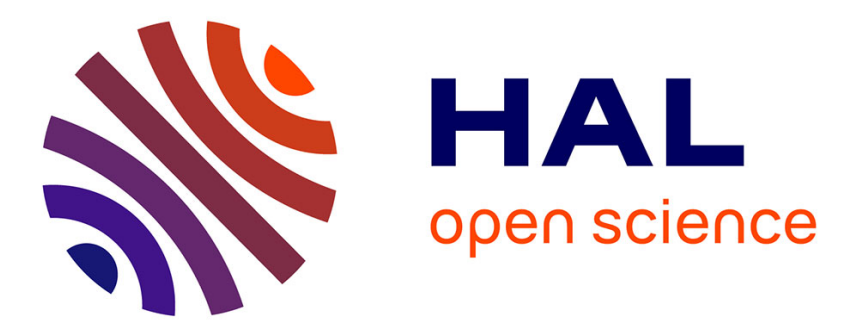

\title{
Co-design of an adaptive hyperspectral imager based on MEMS arrays: from proof of principle to a research prototype
}

\author{
Julien Roul, Frédéric Blanc, Simon Lacroix, Antoine Monmayrant
}

\section{- To cite this version:}

Julien Roul, Frédéric Blanc, Simon Lacroix, Antoine Monmayrant. Co-design of an adaptive hyperspectral imager based on MEMS arrays: from proof of principle to a research prototype. Symposium on Design, Test, Integration \& Packaging of MEMS and MOEMS (DTIP), May 2017, Bordeaux, France. 4p. hal-01522330

\section{HAL Id: hal-01522330 \\ https://hal.science/hal-01522330}

Submitted on 14 May 2017

HAL is a multi-disciplinary open access archive for the deposit and dissemination of scientific research documents, whether they are published or not. The documents may come from teaching and research institutions in France or abroad, or from public or private research centers.
L'archive ouverte pluridisciplinaire HAL, est destinée au dépôt et à la diffusion de documents scientifiques de niveau recherche, publiés ou non, émanant des établissements d'enseignement et de recherche français ou étrangers, des laboratoires publics ou privés. 


\title{
Co-design of an adaptive hyperspectral imager based on MEMS arrays: from proof of principle to a research prototype
}

\author{
Julien Roul $^{1}$, Frédéric Blanc ${ }^{1}$, Simon Lacroix ${ }^{1}$, Antoine Monmayrant ${ }^{1, *}$ \\ ${ }^{1}$ LAAS-CNRS, Université de Toulouse, CNRS, Toulouse, France \\ *Corresponding author: antoine.monmayrant@laas.fr
}

\begin{abstract}
We have defined a hyperspectral imager based on micro-mirror MEMS array, that features easily adaptable spectral resolution, adjustable acquisition time, and high spatial resolution independent of spectral resolution. We present the development of an integrated research prototype of the imager, that allows the control of both the micro-mirrors array and CMOS data reading from the same FPGA board, so as to allow the definition of acquisition schemes adapted to the scene in real time.
\end{abstract}

\section{INTRODUCTION}

Traditional hyperspectral imagers adopt a scanning process to acquire three dimensions of data (two spatial and one spectral which constitute a "hyperspectral cube") on a 2D imager. Not only this constrains the acquisition process, but it also generates high volume of data to process, a large part of it being useless in most of the applications.

Various approaches have been proposed to fit all the information in the hyperspectral cube onto the 2D detector, allowing real-time hyperspectral acquisition [1]. However, most of these approaches rely on a complex and fixed optical system that interweaves spectral and spatial informations. They usually rely on a resource-intensive post-processing to retrieve the hyperspectral cube, which is hence not available in realtime, prohibiting their application in numerous applications (e.g. autonomous mobile systems). Moreover, these systems still generate the full hyperspectral cube, and their characteristics (in particular spectral and spatial resolution) are fixed by design.

We have recently developed an adaptive hyperspectral imager based on a co-designed optical setup with a micromirror MEMS array [2]. We present here the realization of a prototype of this imager, that allows the development of a variety of tightly interleaved acquisition control and data processing algorithms, so as to satisfy the requirements of a large spectrum of applications.

\section{DUAL DiSPERSER IMAGER}

The proposed system is comprised of two gratings (or prisms) separated by a spatial light modulator (SLM). The first half of the setup is a 4f-line [3] centered around a grating which forms a spectrally dispersed image on the SLM. Figure 1 illustrates the case in which the SLM allows only a slice of the dispersed image to pass through into the second half of the setup which is another 4f-line ending with the detector. The purpose of the second grating is to undo the spectral spreading inroduced by the first one, allowing for an undispersed image at the detector. With this setup, the spectral components selected by the SLM are placed at their original spatial positions relative to each other, thus reconstructing a spectrally filtered image. If all of the light is transmitted by the SLM then an undispersed, unfiltered image is formed.

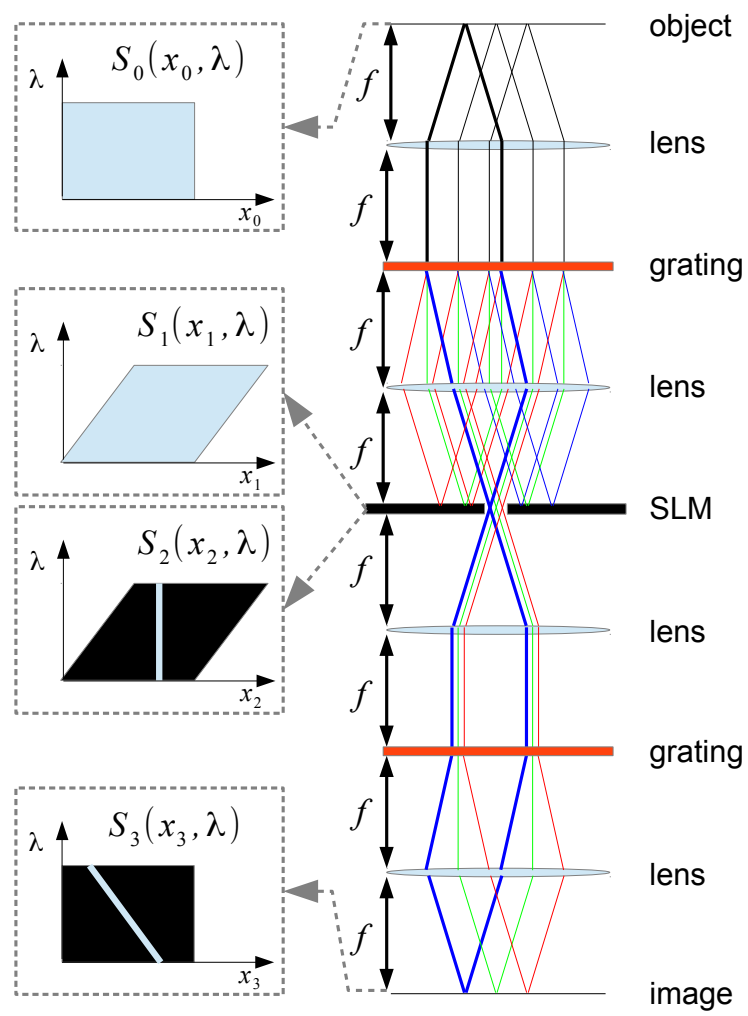

Fig. 1: Proposed setup (right), and illustration of the spectral selection done at the output of the SLM and on the detector, in case the SLM only opens a slit. $f$ is the focal length of the lenses. The spectral density at the source, just before and after the SLM and at the detector are represented by $S_{0}, S_{1}$, $S_{2}$, and $S_{3}$, respectively. 
A precise geometrical optics analysis and a Gaussian optics modelling of the system is proposed in [2]. The main characteristics of the system are:

- When opening a single slit on the SLM, the recorded image gives a direct access to a 2D subset of the hyperspectral cube that corresponds to a slanted cut inside the cube along the spectral direction. The dual disperser acts as a narrow band spectral filter with a linear gradient in wavelength along one spatial direction, and the position of the slits on the SLM determines the offset of this gradient.

- All the spectral components of the hyperspectral cube sharing the same spatial position are co-located at the same position on the image. In other words, there is no wavelength dependent spatial shift on the image: the position at which we retrieve an element from the hyperspectral cube on the image does not depend on its wavelength coordinate in the cube.

- The spatial resolution of the system does not depend on the parameters which determine spectral resolution (i.e. slit width, grating periodicity, and focal length). The spectral resolution is given by the difference in wavelength corresponding to two neighboring slit positions at any single point on the detector. The dependence of the spectral resolution on slit width and dispersion is the same as that of a conventional spectrometer.

These properties are all modeled with simple linear equations, and imply that there is no need for a complicated reconstruction algorithm to recover the spatial coordinates of points in the hyperspectral cube.

\section{DATA ACQUiSITION SCHEMES}

In a nutshell, the system acts as a programmable, spatially dependent, spectral filter for which spatial resolution is independent of spectral resolution and for which there is no wavelength dependent shift of the image at the detector. This opens for a variety of data acquisition schemes, either preprogrammed or adapted in real-time to the spatial and spectral content of the imaged scene.

\section{A. Systematic scanning}

Using a simple slit pattern, the whole hyperspectral cube can be readily retrieved by scanning the slit across the SLM and recording the images corresponding to each slit position: with respect to usual spatial or spectral scanning processes, here the scanning is done with slanted sections of the cube. This acquisition scheme offers limited advantage over classic hyperspectral imagers (no moving parts), as the acquisition takes time and produces a full hyperspectral cube, like in classical hyperspectral imagers.

\section{B. Random access monochromatic imaging}

By synchronizing the acquisition of image columns with the opening of a slit along the columns of the SLM, one can readily recover a monochromatic image, at a wavelength that is trivially defined by the relative positions of the acquired and opened columns. This allows the acquisition of a sequence of arbitrary-wavelength monochromatic images at video rate. With rolling shutter cameras for instance, this acquisition process can be achieved by synchronizing the slit openings on the SLM with the image lines readout frequency.

\section{Near Snapshot Partitioning}

The system properties allows the definition of an adaptive acquisition scheme that allows to extract the spectral content of uniform intensity regions. The starting point is to assume that there exist in the hyperspectral cube spatial regions that are spectrally homogeneous. This is a reasonable sparsity assumption that corresponds to most realistic situations, where the spectral content is strongly spatially correlated. One possible way to identify these regions is to look for contiguous regions of iso-intensity on the unfiltered image obtained with a fully transmissive SLM. Assuming such regions are spectrally homogeneous, the spatial coordinates in the region can be used to encode different spectral channels.

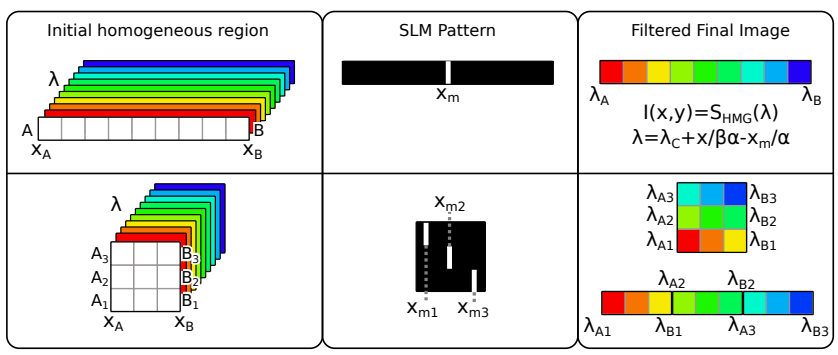

Fig. 2: Snapshot partitioning: in spectrally homogeneous regions, the spatial coordinates in the final image can be used to encode all the spectral channel by a simple choice of multi-slit pattern to apply on the SLM.

A canonical example of a homogeneous region is presented on figure 2 (top): it is one horizontal segment of pixels $[A, B]$ along the $x$ direction, $x_{A}$ and $x_{B}$ denoting the starting and ending $x$-coordinate of this segment. For a simple slit pattern programmed on the SLM at the position $x_{m}$, the intensity $I(x, y)$ on the final image along this segment corresponds to the spectral density, expressed by a simple linear relation which is a function of the the relative slit and pixel position and some calibration parameters. If the homogeneous region is wide enough, its whole spectral density can be retrieved in a single acquisition. If not, its height can be exploited to extend this range, as illustrated Figure 2 (bottom).

So by taking an unfiltered initial image, partitioning it in homogeneous regions of uniform intensity and assuming they have the same spectral content, simple geometric reasoning can be exploited to automate the extraction of their spectral content.

\section{Compressed sensing}

The dual disperser described here is extremely similar to the CASSI[4] that was introduced as a compressed sensing hyperspectral imager. Compressed sensing is a way to produce detailed reconstructions from a limited number of 
measurement by exploiting sparcity in the signal to be reconstructed. In the field of hyperspectral imaging, spatially and spectrally homogeneous regions (fairly common in most use cases of hyperspectral imaging) can be harnessed to implement compressed sensing acquisition schemes. Although it allows real-time acquisition of the data, compressed sensing relies on a long and resource-intensive reconstruction step. With our new prototype, new acquisitions for varying micro-mirror arrangement can be made during the reconstruction step and can be harnessed to speed up the identification of the best reconstruction estimate.

\section{PRototy Pe DESIGN}

In order to implement the acquisition schemes proposed above a new prototype had to be envisioned. On the optical side, a new setup (see Figure 3) has been designed to minimize optical aberrations and optimize the matching between the CMOS imager size and pixel size with the one of the SLM.

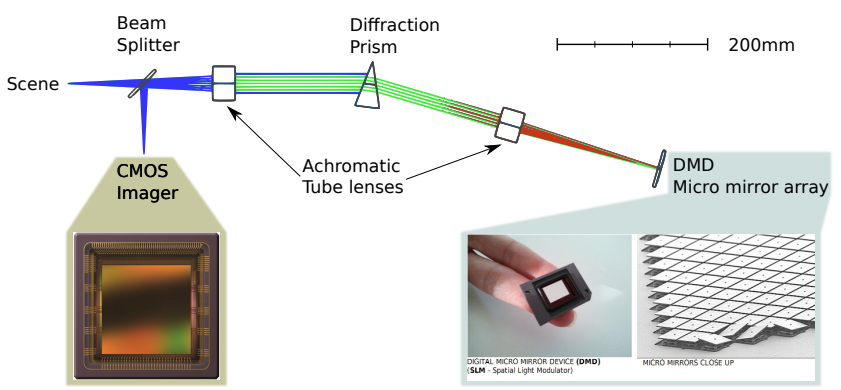

Fig. 3: Optical design for the new prototype based on a dispersion prism and achromatic tube lenses.

The CMOS dectector (CMOSIS CMV4000) is a $2048 \times$ 2048 matrix of $5.5-\mu \mathrm{m}$-size pixels, offering a square field of view of $11 \times 11 \mathrm{~mm}^{2}$. For the SLM, a DMD micromirror array (Texas Instruments DLP9500BFLN) with $1920 \times 108010.8$ $\mu \mathrm{m}$-wide-micro-mirrors offers a surface of $20.7 \times 11.7 \mathrm{~mm}^{2}$, enough to accommodate the same field of view than the CMOS detector with some extra width to account for the diffraction.

The optical arrangement was optimized to reduce chromatic aberrations by using diffraction-limited achromatic lenses. These are 200-mm-focal-length tube lenses (Thorlabs TTL200) usually used in microscopes to image the field of view on a CMOS or a CCD. For the dispersion, a custommade, $30^{\circ}$-apex, prism was chosen in place of a diffraction grating. Indeed, it proved impossible to get a diffraction grating that, once combined with the $200-\mathrm{mm}$ lenses, would provided a suitable dispersion without introducing unwanted diffraction order.

To compensate for the wavefront tilt introduced by the DMD micromirror array, the imager was tilted, reducing optical aberrations, as can been seen on figure 4. Using ray tracing, we simulate the propagation inside the optical system for an observed scene containing three spatially overlapping grids at three different wavelengths of $\lambda_{b}=450 \mathrm{~nm}$ (blue), $\lambda_{g}=550 \mathrm{~nm}$ (green) and $\lambda_{r}=650 \mathrm{~nm}$ (red). The intermediate image formed onto the DMD (figure 4 (right)) clearly shows

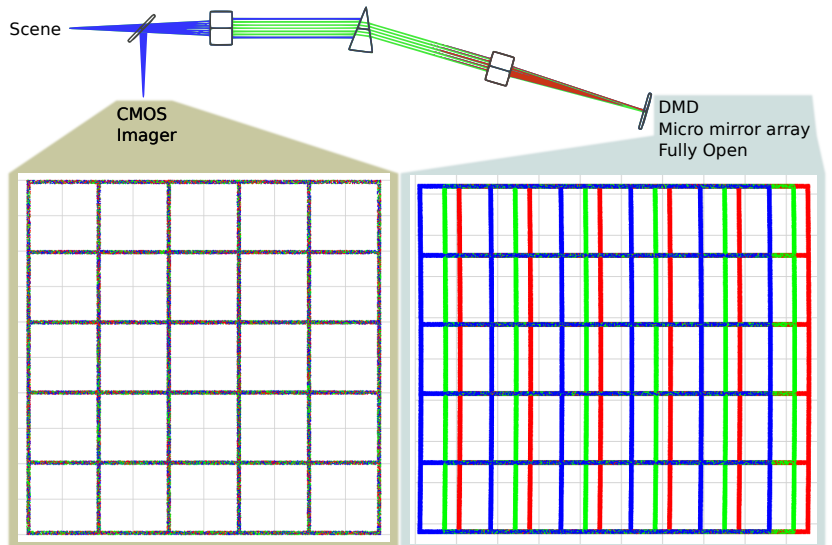

Fig. 4: Optical simulations (ray tracing): view of the final image on the CMOS detector (left) and of the intermediate image on the DMD (right) for a fully open DMD and a scene under observation made of three monochromatic grids at $\lambda_{b}=$ $450 \mathrm{~nm}$ (blue), $\lambda_{g}=550 \mathrm{~nm}$ (green) and $\lambda_{r}=650 \mathrm{~nm}$ (red).

the spectral separation off the three grids. One can also observe a slight distortion of the grids, the vertical segments being slightly curved. This distortion is introduced by the single pass inside the prism. We assume for this simulation that the DMD is fully transmissive (all the spectral components are reflected), which should result in the formation of a panchromatic image on the CMOS. The final image on the CMOS detector (figure 4left) shows a well resolved panchromatic image, with the three spectral grids well overlapped again. Moreover, thanks to a second pass inside the same prism, the curve distortion is compensated for. To determine the potential spectral resolution, a second ray tracing simulation was carried out: for a DMD with only one vertical slit transmissive, the rest of the DMD rejecting the incoming light. Moreover, instead of three monochromatic grids, three monochromatic uniform squares were used as an observed scene. As can be seen on figure 5, we obtain three vertical lines in the final image, one for each monochromatic plane. The overall distance between the two extreme lines (above 220 pixels) gives an idea of the achievable spectral resolution.

With the current combination of prism and lenses, a 200$\mathrm{nm}$-wide spectrum covering most of the visible is spread over more than 220 pixel on the CMOS detector allowing to resolve a maximum of around 200 spectral bands, without any issues of unwanted diffraction orders.

To allow precise timing and closed-loop control of the whole system, both the CMOS imager and the micro-mirror array are controlled by the same FPGA board (Vialux VX4100), shown on Figure 6. This FPGA, initially controlling the micro-mirror array alone has all the required interfaces and processing capabilities to also control the CMOS imager. In particular, the combination of the CMOS and FPGA we have selected allows fast and easy retrieval of image subsets, required for an efficient implementation of the random access monochromatic imaging scheme described above. 


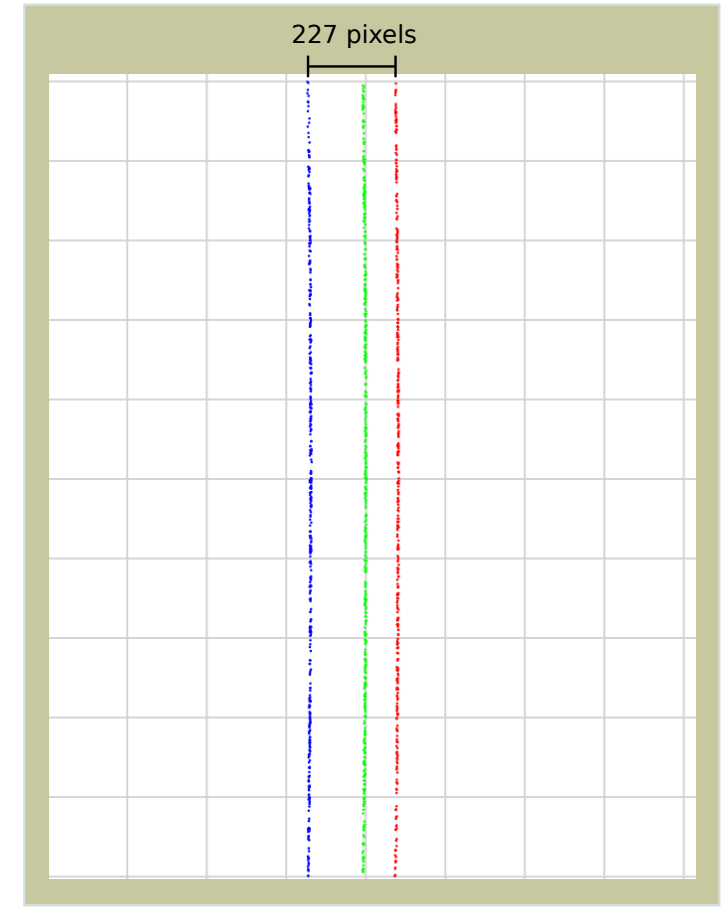

Fig. 5: Optical simulations (ray tracing): final image on the CMOS for three uniformly lit monochromatic squares at $\lambda_{b}=$ $450 \mathrm{~nm}$ (blue), $\lambda_{g}=550 \mathrm{~nm}$ (green) and $\lambda_{r}=650 \mathrm{~nm}$ (red) and a DMD with one single vertical line transmitting.

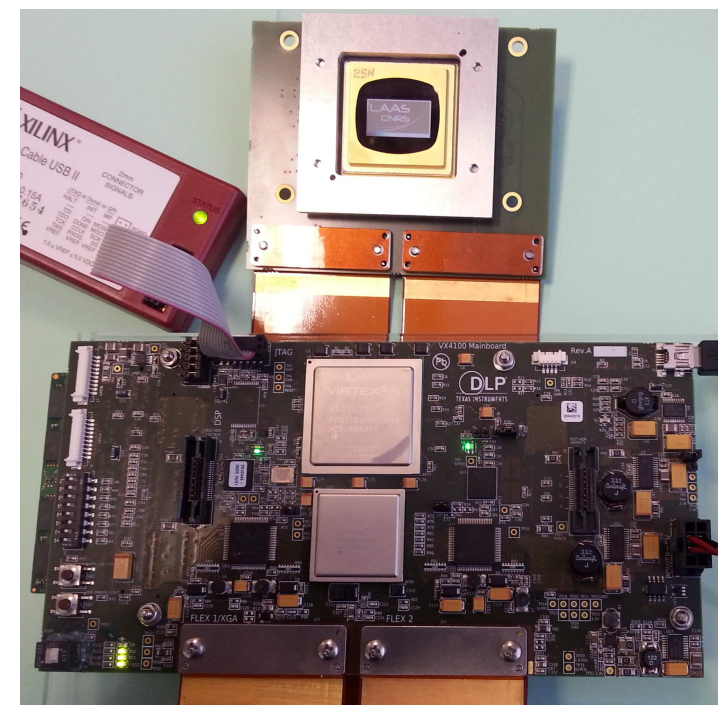

Fig. 6: FPGA board for tight loop control of both CMOS detector and micro-mirror array.

\section{CONCLUSion}

We have presented the principle of an adaptive hyperspectral imager, that yields the possibility to define a variety of acquisition schemes, either tailored to some a priori objectives or dynamically adapted to the characteristics of the perceived scene. In both kind of acquisition schemes, a tight coupling between the DMD control and data acquisition is required, which calls for a tight integration of the DMD and camera drivers and the algorithms that define the acquisition. For this purpose we have selected a FPGA hardware architecture able to embed both the devices control and the data processing algorithms. From the optical point of view, we dimensioned both the focal length of the lenses and the dispersion of the prism in order to adapt the numerical aperture to the array and pixel sizes of both the DMD and the camera and to optimize the spectral spreading on the DMD and thus the spectral resolution of the prototype. A particular care was taken to reduce chromatic aberration and wavefront tilt.

\section{REFERENCES}

[1] Nathan Hagen and Michael W Kudenov. Review of snapshot spectral imaging technologies. Optical Engineering, 52(9):090901-090901, 2013.

[2] Scot McGregor, Simon Lacroix, and Antoine Monmayrant. Adaptive hyperspectral imager: design, modeling, and control. Journal of Optics, 17(8):085607, 2015. doi: 10.1088/2040-8978/17/8/085607.

[3] Antoine Monmayrant, Sébastien Weber, Béatrice Chatel, et al. A newcomer's guide to ultrashort pulse shaping and characterization. Journal of Physics B: Atomic, Molecular and Optical Physics, 43(10), 2010.

[4] ME Gehm, R John, DJ Brady, RM Willett, and TJ Schulz. Single-shot compressive spectral imaging with a dualdisperser architecture. Optics Express, 15(21):1401314027, 2007. 